\title{
Epidermal Layers Characterisation by Opto-Magnetic Spectroscopy Based on Digital Image of Skin
}

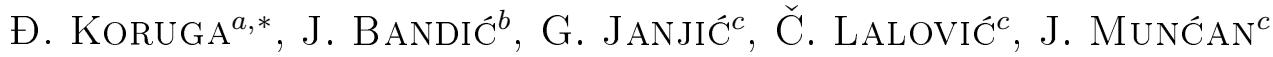 \\ AND D. Dobrosavljević Vukojević ${ }^{d}$ \\ ${ }^{a}$ NanoLab, Faculty of Mechanical Engineering, University of Belgrade \\ Kraljice Marije 16, Belgrade, 11120/35, Serbia \\ ${ }^{b}$ ORS HOSPITAL Belgrade, Milovana Marinkovića 23, Belgrade, 11000, Serbia \\ ${ }^{c}$ Biomedical Engineering, Faculty of Mechanical Engineering, University of Belgrade \\ Kraljice Marije 16, Belgrade, 11120/35, Serbia \\ ${ }^{d}$ Clinic of Dermatovenerology, School of Medicine, University of Belgrade, Pasterova 2, 11000 Belgrade, Serbia
}

(Received March 21, 2011)

\begin{abstract}
According to the most literature data, the skin is usually observed as a simple structure with equivalent electrical model, which includes general properties of epidermis, basal membrane and dermis. In this paper, we analyzed the skin structure as a more complex system. Particularly we analyzed epidermis based on layers approach and its water organization in lipids ordered in sub-layers. Using opto-magnetic spectroscopy method, which is very sensitive to paramagnetic/diamagnetic properties of the tissue, we found out that nanowater structure ordering in lipids of epidermal layers play very important role in skin properties. We use bioimpedance as complementary and compatible method to opto-magnetic spectroscopy in skin characterization. In our investigation we found out the difference of the skin properties of the people who are drinking two different type of water ( $\mathrm{Z}$ and $\mathrm{N})$. We observed the significant difference in middle part of stratum granulosum, where water-lipid sub-layers exists. These results indicate importance of water nanolayers presence in epidermis and type of drinking water reflecting on human skin properties.
\end{abstract}

PACS: 43.58.Bh, 07.05.Pj, 42.79.-e, 42.30.-d, 42.25.Ja, 87.64.Dz

\section{Introduction}

There are three main different skin layers: epidermis, dermis, and subcutis. Between epidermis and dermis, there is a very thin layer known as basement membrane, which structurally and energetically separates them. Because these layers are composed of different types of cellular and extracellular molecules, they exhibit different types of light propagation.

The epidermis is thick about $200 \mu \mathrm{m}$ on average and its thickness varies depending on the location on the body, and can be up to $2 \mathrm{~mm}$ thick. However, the thickness also varies according to the volume of the water that epidermis holds [1-3].

The epidermis is anatomically divided into five sublayers: stratum corneum (horny cell layer), stratum lucidum (clear layer - can only be found in soles and palms), stratum granulosum (granular layer), stratum spinosum (prickle cell layer) and stratum basale (basal cell layer) (Fig. 1). The epidermis is metabolically active tissue. One type of epidermal cells, keratinocytes, move upward to the outer surface. This process is called turn-over, and

\footnotetext{
* corresponding author; e-mail: dkoruga@mas.bg.ac.rs
}

takes about 28 days (maximum 72 days). During this process keratinocytes change their structure and physiological function [4].

Keratinocytes are produced in the stratum basale which holds approximately $10 \%$ of the epidermal water. With aging, this layer becomes thinner and loses the ability to retain water. Basal cells, through the process of turn-over, make their shape somewhat flatter and form stratum spinosum layer with about 20 layers that lie on the top of the basal cell layer. The thickness of the stratum spinosum layer is from 60 to $150 \mu \mathrm{m}$, and holds about $35 \%$ of epidermal water. In the next turn-over process organelles such as nuclei and mitochondria start to resolve. Cells are increasingly filled with keratin fibers and contain less intracellular water than basal and spinosum cells. However, this layer called stratum granulosum, is about $5 \mu \mathrm{m}$ thick and has very well ordered lipid-water layers, from 5 to 20 , depending on skin conditions. Water layers are thin from 20 to $50 \mathrm{~nm}$ (Fig. 2, left).

In this study we used the combination of two different methods in order to investigate skin layers properties. One of them was opto-magnetic spectroscopy (OMS), a novel method in investigating surface of matter and optical and magnetic properties of thin layers of mate- 


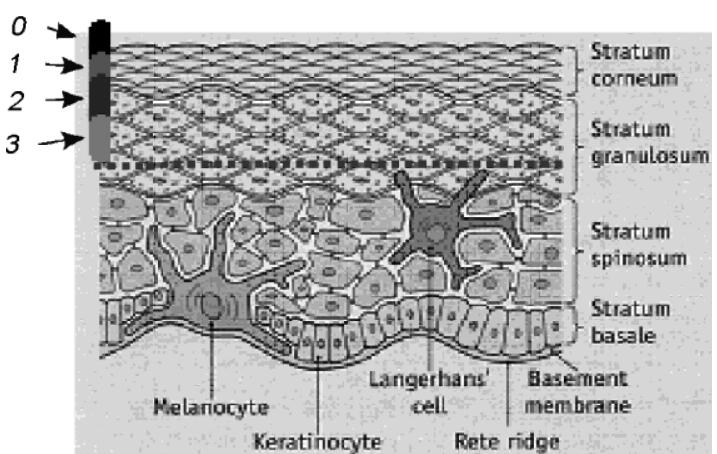

Fig. 1. Cross-sectional anatomy of the epidermis with four main layers and basement membrane and other structures like melanocyte, Langerhans cell and etc. (adapted from [2]). Left numbering notation indicates: 0 - skin surface with impurities, surface oiliness, surface moisture and first level of stratum corneum without water (about $6 \mu \mathrm{m}), 1$ - deeper part of stratum corneum with very small amount of water (about $5 \mu \mathrm{m}$ ), 2 - water in stratum corneum and the first water layers in stratum granulosum with significant amount of water (from $10 \mu \mathrm{m}$ to $15 \mu \mathrm{m}$ ), 3 - stratum granulosum, rich with water - if the skin is in good condition (about $20 \mu \mathrm{m})$. Stratum granulosum holds water in water/lipid layers, and only in this part of the skin it is possible to characterize the water as an independent substance (see Fig. 2, picture on the left).

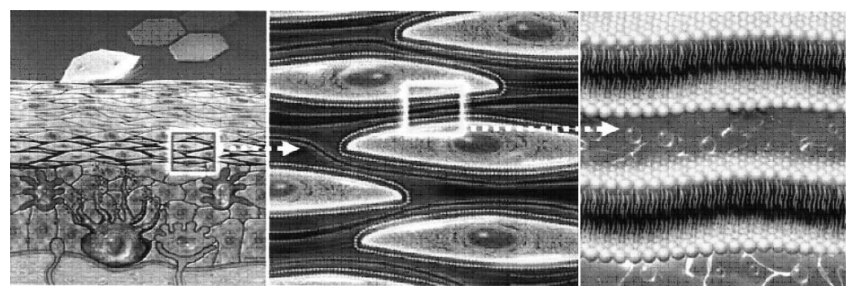

Fig. 2. Epidermis of human skin (left) and its layers (adapted from [2]). Square in first picture indicates stratum granulosum, which is magnified in the second picture (middle), while third picture (right) shows lipid layers and water between them. Thickness of the water layer is between 20 and $50 \mathrm{~nm}$, giving layer properties as liquid crystalline water. Water is well ordered in the form of quasi-polymer state.

rials and tissues. Method is based on the difference between responses of the material when it is illuminated with both white light under right angle (reflected light to sensor is diffuse) and with the same white light under the Brewster angle (reflected light to sensor is polarized). Second method, complementary and compatible to OMS is bioimpedance.

\section{Material and methods}

We investigated human epidermal layers properties for two different types of drinking waters: $\mathrm{N}$ - normal tap water, and $\mathrm{Z}$ - tap water, which contains four times more ortophosphates (contents $0.64 \mathrm{mg} / \mathrm{l}$, allowed $0.15 \mathrm{mg} / \mathrm{l}$ ), twice more residual chlorine (contents $1.00 \mathrm{mg} / \mathrm{l}$, allowed $0.5 \mathrm{mg} / \mathrm{l}$ ) and 1.70 more iron (contents $0.51 \mathrm{mg} / 1$, allowed $0.3 \mathrm{mg} / \mathrm{l}$ ). We use fifteen volunteers who have been drinking $\mathrm{Z}$ water for years and five people who are drinking $\mathrm{N}$ water. For all of them we characterized skin surface (inner arm and forehead) by OMS and bioimpedance, then we removed all the impurities from surface skin (dust, surface oil, surface water, etc.) by sticking plaster (step 0 in Fig. 1) and characterized first layer of the stratum corneum by both methods. Then, we used sticking plaster and characterized the first layer of the stratum granulosum, then we removed the first half of the stratum granulosum and characterized it, and finally we removed second half part of the stratum granulosum and characterized water in lipid-water layers. From the day of experiment to the next six weeks, ten volunteers who drank $\mathrm{Z}$ water, started to drink $\mathrm{N}$ water. After eight weeks we made epidermis characterization by the same procedure as previous one. Five people who were drinking $\mathrm{N}$ water and five people who were continuously drinking $\mathrm{Z}$ water became control group. In our experiment, we had volunteers of different age (from 11 to 63 ), gender ( 8 male and 12 female) and skin type 2 and 3 . The participants gave their informed consent prior to experiment. Relative humidity and room temperature were $48 \pm 0.2 \%$ and $22 \pm 0.3^{\circ} \mathrm{C}$, respectively.

OMS technique is based on electron properties of matter (covalent bonds, hydrogen bonds, ion-electron interaction, van der Waals interaction) and its interaction with light [5]. The method was originally developed for early skin cancer and melanoma detection by MySkin, Inc., USA [6, 7]. OMS method has been used for characterization of blood plasma [8], contact lenses [9], and water [10]. Algorithm for data analysis is based on chromaticity diagram called "Maxwell's triangle" and spectral convolution operation according to ratio of $(R-B)$ and $(W-P)$, where: $R$ - red channel, $B$ - blue channel, $W$ - white diffuse light, and $P$ - reflected (under the Brewster angle) polarized white light (6). The abbreviated designation means that $(R-B)$ is (red minus blue wavelength) of $(W-P)$ (image of white light minus image reflected polarized light) are used in spectral convolution algorithm to calculate data for opto-magnetic spectroscopy of the matter.

Bioimpedance measurement has been done by BIA-1 (NanoLab, University of Belgrade) analyzer on 9, 30, 47, and $100 \mathrm{kHz}$, with input voltage from $1.5 \mathrm{~V}$ to $5.0 \mathrm{~V}$ (peak-to-peak), by two electrodes (stainless steel, diameter $10 \mathrm{~mm}$, distance between electrode centers is $30 \mathrm{~mm}$ ).

Thickness of removed skin layers on sticker plaster surface was investigated by atomic force microscopy (AFM) (NanoProbe JEOL JSPM 5200, Japan).

\section{Results and discussion}

Skin properties for inner arm region of five volunteers, who drank N (normal) water have shown similarity of convolution spectra for wavelength difference on 
$132 \pm 1.2 \mathrm{~nm}$. However, five volunteers who drank water $\mathrm{Z}$ all the time, possess peak on $140 \pm 1.2 \mathrm{~nm}$. In ten volunteers who switched drinking water (from $\mathrm{Z}$ to $\mathrm{N}$ ) for two months, there was peak on $135 \pm 1.5 \mathrm{~nm}$. Wavelength shift difference of volunteers who drank $\mathrm{N}$ water and $\mathrm{Z}$ water was maximum $10.4 \mathrm{~nm}$ and minimum $5.6 \mathrm{~nm}$. Convolution spectra of volunteers, who changed type of drinking water for two months, had values between $\mathrm{Z}$ and $\mathrm{N}$ volunteers' spectral data. This indicates that skin (dermis and subcutis) holds water longer than two months. We assume that the water in dermis of these volunteers was the mixture of water type $\mathrm{N}$ and $\mathrm{Z}$ and penetrates through basement membrane to epidermis. In the future experiments, based on the wavelength difference shift, we will test how long the water stays in the human skin.

Here, we present the skin opto-magnetic spectra for two males, age 11 (Fig. 3a-d) and age 63 (Fig. 4a-d) for inner arm region. There is difference of the skin property for each epidermal layer. However, peak on $132 \pm 1.5 \mathrm{~nm}$ wavelength difference exists in both cases. Volunteers who switched to drink water (from Z to $\mathrm{N}$ ) for two months, showed difference in peaks from $120 \mathrm{~nm}$ to $130 \mathrm{~nm}$ comparing to volunteers who drank $\mathrm{N}$ or $\mathrm{Z}$ water (Figs. 5 and 6). OMS spectra difference has been observed for both regions: inner arm and forehead. However, more pronounced difference has been observed for forehead than inner arm. We assume that the reason was more complex skin structure of the forehead (presence of sebaceous glands and the skin was moist and more oily).
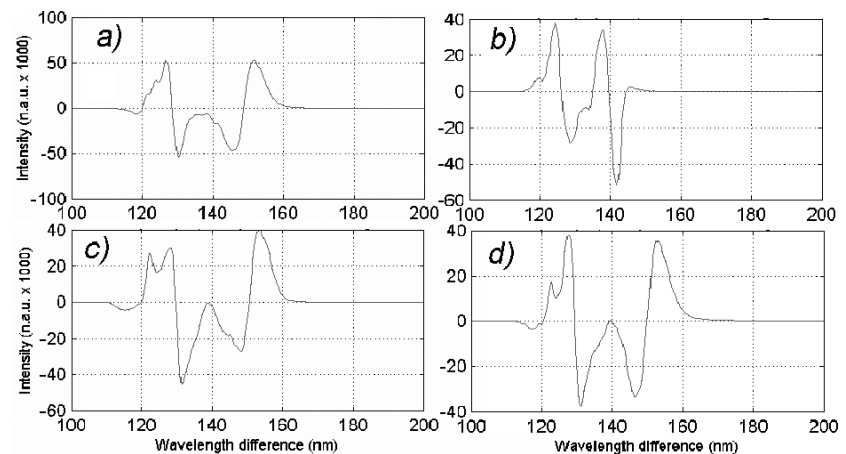

Fig. 3. Case 1: Optomagnetic spectroscopy of skin layers, inner arm region, from male aged 11 years: (a) spectra for every day skin surface, (b) spectra of the skin when impurities, surface water, surface oiliness and first stratum corneum cells were removed, (c) spectra of the skin when stratum corneum was removed, (d) spectra of the skin when about $50 \%$ of cells of stratum granulosum were removed (see Fig. 1).

The interaction of lipids and water are fundamental to all body tissues, but for skin it has special significance. Each water molecule is able to hydrogen bond to four neighbors. Water hydrogen bonds make network with the popular head groups of lipids. The lipids of stratum corneum consist mainly of ceramides, cholesterol, and fatty acids. On skin surface polar lipids are capable
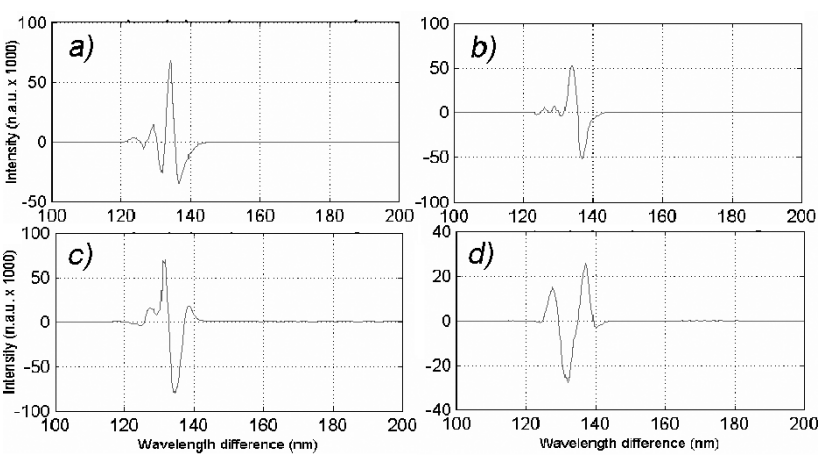

Fig. 4. Case 2 - as in Fig. 3, but for male aged 63.
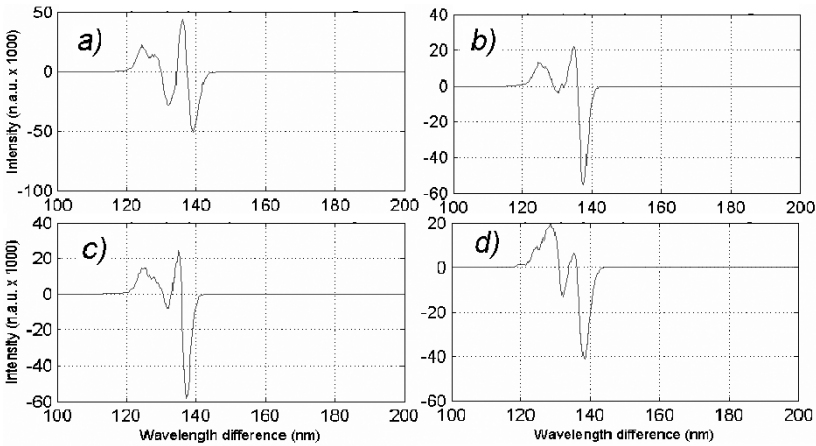

Fig. 5. Case 3 - as in Fig. 3, but for male aged 50.

to form lamellar or hexagonal phases in the presence of excess water. A liquid ordered phase has both properties of a gel phase and a liquid crystalline phase. The phase's mixture and properties of skin layers are depending on many factors but three are dominant: age, gender, and skin type. Removed skin layers were characterized by AFM (Fig. 7) and their thickness was measured for inner arm.

When we compare all cases for layer 2, where skin holds water in water-lipid layers, we find out difference for volunteers who drank water type $\mathrm{N}$ and Z (Fig. 8). Peak of wave length difference between $120 \mathrm{~nm}$ and $130 \mathrm{~nm}$ goes up (Fig. 8a and b), while for volunteers who change type
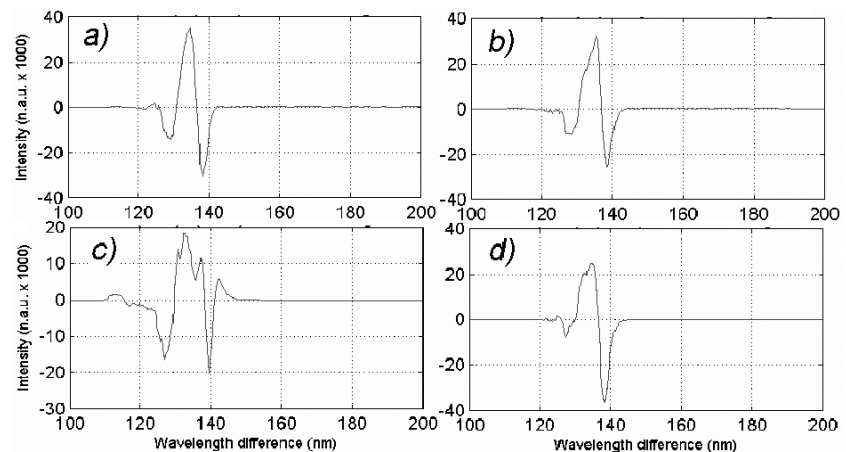

Fig. 6. Case $4-$ as in Fig. 3, but for male aged 43 . 
of drinking water for two months (from $\mathrm{Z}$ to $\mathrm{N}$ ) peak goes up and then down (Fig. 8c). However, in case for volunteers who drank water $\mathrm{Z}$ all time peak goes down (Fig. 8d). For the same group of drinking water (except for age 11) bioimpedance shows small difference for all volunteers for layers 0 and 1, while for layers 2 and 3 show significant difference for water type $\mathrm{N}$ and $\mathrm{Z}$ (case 3 - change drinking water from $\mathrm{Z}$ to $\mathrm{N}$, and case 4 all time drinking water $\mathrm{Z}$ ).

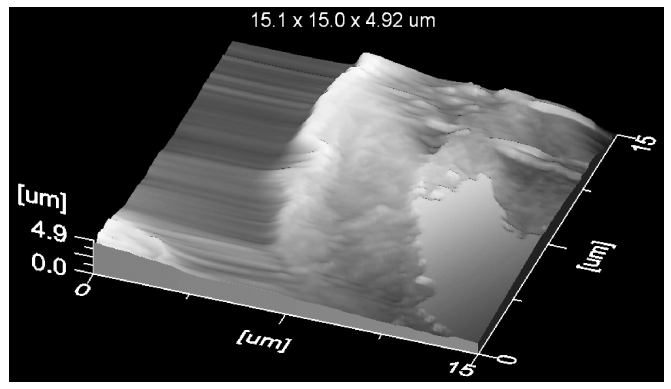

Fig. 7. Skin removed layer 3 (on sticker plaster surface) characterized by AFM. Removed layer thickness for inner arm (case 4) was $4.92 \mu \mathrm{m}$ (maximal thickness for layer 1 was $10.2 \mu \mathrm{m}$, while the minimal was $4.2 \mu \mathrm{m}$ for layer 0). Maximum removed thickness of all four layers for inner arm and forehead was $36.2 \mu \mathrm{m}$ and $52.8 \mu \mathrm{m}$, while minimum was $30.4 \mu \mathrm{m}$ and $43.6 \mu \mathrm{m}$, respectively.
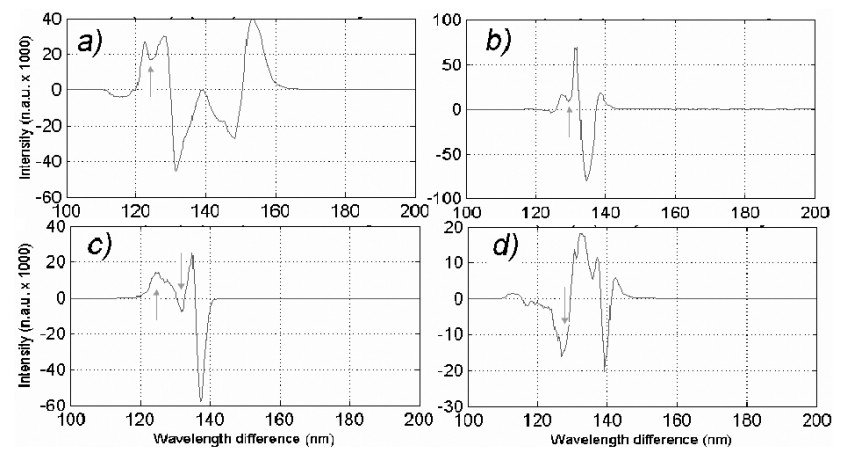

Fig. 8. Opto-magnetic spectroscopy of skin layers, inner arm region, for cases 1-4: spectra present skin properties when both complete stratum corneum and about $20 \%$ of cells of stratum granulose were removed. In region from $120 \mathrm{~nm}$ to $130 \mathrm{~nm}$ wavelength difference peaks were similar: for case 1 and 2 goes up (water $\mathrm{N}$ for all time), for case 3 partially peak goes up and then goes down (change water $\mathrm{Z}$ to water $\mathrm{N}$ for two months), and case 4 , peak goes down (water $\mathrm{Z}$ for all the time).

Bioimpedance measurements show difference of skin layers for young skin (age 11) and old skin (age 63). The old skin (age 63) does not hold water in epidermis well. Dramatic situation is in stratum granulosum layer where difference of young and old skin was dramatically significant (Fig. 8). The young skin, age 11, holds well water in all epidermal layers. On skin surface, impendence is approximately the same for young and old skin. The dif-

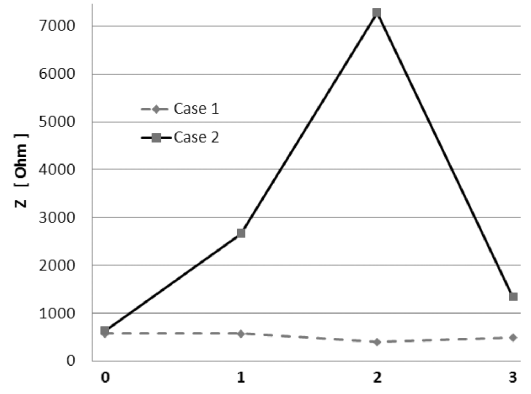

Fig. 9. Bioimpedance $(100 \mathrm{kHz})$ of skin layers, inner arm region, for cases 1 (aged 11) and case 2 (aged 63): significant difference of bioimpedance was found out when complete stratum corneum and about $30 \%$ of cells of stratum granulosum were removed by adhesive bandage. This is the same result which was found out by OMS spectra for region of wavelength difference (Fig. 3 and Fig. 4). In general, old skin (age 63) does not hold water in epidermis well, and in particularly in stratum granulosum layer. However, young skin of age 11 is of much better order and holds water well in all epidermal layers.

ference becomes obvious for stratum corneum, because this layer contain $8 \%$ of all water in epidermis. Also, this result indicates role of a single gel phase in stratum corneum [11]. The gel phase domains could support barrier function, whereas the continuos liquid crystalline domain could provide the flexibility necessary for pliable skin [12].

An important function of the skin is protection against the loss of water. Transdermal water loss (TEWL) is a process of passive diffusion through the skin. The horny layer is the most important rate-limiting step for the transport of the water to the exterior. The unique organization of the hydrophilic cells within the lipid, hydrophobic environment makes this 10 to $20 \mu$ m layer extremely efficient as a barrier.

Today there is evidence that water held in epidermis is reduced in aged individuals compared with TEWL values from mid-adulthood [13]. We found out that significant difference of water presence is in stratum granulosum (Fig. 9). From Fig. 9 one can see that stratum corneum has also water holding capacity (layer 1 : case 1 and case 2) [14].

\section{Conclusion}

Results of the investigation of human skin properties, based on twenty volunteers, using OMS methods, show that bigger difference of spectra is for forehead than for inner arm region. We assume that reason for this evidence lies in the sebaceous gland presence and activity in forehead and its absence in the inner arm region.

For regions, inner arm and forehead, layer obtained when complete stratum corneum and about $50 \%$ of cells of stratum granulosum were removed was more interesting to investigate water in the skin. For these regions, 
both OMS spectra and bioimpedance show skin property difference for two different types of drinking water. This strongly indicates that type of drinking water has influence on skin properties. Characterizing epidermal skin layers by OMS spectra and bioimpedance can establish biological value of drinking water in the skin.

Since OMS method may well determine paramagnetic/diamagnetic properties of tissue, we consider that this method and molecular level approach to investigate skin property is very promising field for basic research (dermatology) with direct influence on application in biomedicine. To use OMS method in cosmetology [15] and for early skin diseases detection and characterization, we plan to do more research in future.

\section{Acknowledgments}

This research has been partially funded by Ministry of Science and Technological Development of Republic of Serbia, through project III41006, and partially with resources of MySkin Inc, USA (Patent PCT/US2008/ 050438)

\section{References}

[1] A. Waugh, A. Grant, Anatomy and Physiology, Churchill Livingsone, Edinburgh 2007.

[2] D.J. Gawkrodger, Dermatology, Churchill Livingsone, Edinburgh 2003.

[3] H. Arimoto, M. Egawa, Y. Yamada, Skin Res. Technol. 11, 27 (2005).
[4] Bioengineering of the Skin: Water and the Stratum Corneum, Eds. J. Fluher, P. Elsner, E. Berardeca, H. Maibach, CRC Press, Boca Raton 2005.

[5] Đ. Koruga, A. Tomic, Z. Ratkaj, L. Matija, Mater. Sci. Forum 518, 491 (2006).

[6] Đ. Koruga, A. Tomic, US Pat. App. No. 61/061, 852, 2008, PCT/US2009/030347, Publication No: WO/ 2009/089292, Publication Date: July 16 (2009).

[7] J. Bandic, Đ. Koruga, R. Mehendale, S. Marinkovich, US Pat. App. No. PCT/US2008/050438, Publication No: WO $/ 2008 / 086311$, Publication Date: July 17 (2008).

[8] M. Papic-Obradovic, Đ. Kojic, L. Matija, Acta Phys. Pol. A 117, 782 (2010).

[9] D. Stamenkovic, D. Kojić, L. Matija, Z. Miljkovic, B. Babic, Int. J. Mod. Phys B 24, 825 (2010).

[10] Đ. Koruga, S. Miljkovic, S. Ribar, L. Matija, D. Kojic, Acta Phys. Pol. A 117, 777 (2010).

[11] L.J. Norlen, Invest Dermatol. 117, 830 (2001).

[12] N. Nakagawa, M. Matsumoto, S. Sakai, Skin Research Technology 16, 137 (2010).

[13] J.A. Bouwstra, J. Lipid Res. 42, 1759 (2001).

[14] B.A. Gilchrest, J. Am. Acad. Dermatol. 21, 610 (1989).

[15] L. Norlen, Int. J. Cosmetic Sci. 28, 397 (2006). 